\title{
Assessment of Near-Field Sonic Boom Simulation Tools
}

\author{
J. H. Casper ${ }^{*}$, S. E. Cliff ${ }^{\dagger}$, S. D. Thomas ${ }^{\ddagger}$, M. A. Park ${ }^{*}$, \\ M. S. McMullen ${ }^{\S}$, J. E. Melton ${ }^{\dagger}$, and D. A. Durston ${ }^{\dagger}$
}

A recent study for the Supersonics Project, within the National Aeronautics and Space Administration, has been conducted to assess current in-house capabilities for the prediction of near-field sonic boom. Such capabilities are required to simulate the highly nonlinear flow near an aircraft, wherein a sonic-boom signature is generated. There are many available computational fluid dynamics codes that could be used to provide the near-field flow for a sonic boom calculation. However, such codes have typically been developed for applications involving aerodynamic configuration, for which an efficiently generated computational mesh is usually not optimum for a sonic boom prediction. Preliminary guidelines are suggested to characterize a state-of-the-art sonic boom prediction methodology. The available simulation tools that are best suited to incorporate into that methodology are identified; preliminary test cases are presented in support of the selection. During this phase of process definition and tool selection, parallel research was conducted in an attempt to establish criteria that link the properties of a computational mesh to the accuracy of a sonic boom prediction. Such properties include sufficient grid density near shocks and within the zone of influence, which are achieved by adaptation and mesh refinement strategies. Prediction accuracy is validated by comparison with wind tunnel data.

Nomenclature

$\begin{aligned} & \text { Symbols } & & \underline{\text { Greek }} \\ C_{L} & \text { Lift Coefficient } & \alpha & \text { Aircraft Angle of Attack } \\ C_{P} & \text { Pressure Coefficient } & \phi & \text { Azimuthal Displacement Angle } \\ h & \text { Sampling Distance from Aircraft Surface } & \mu & \text { Mach Angle } \\ L & \text { Aircraft Characteristic Length } & & \\ M & \text { Mach Number } & & \underline{\text { Abbreviations }} \\ P & \text { Aerodynamic Pressure } & \text { CFD } & \text { Computational Fluid Dynamics } \\ \Delta P & \text { Local Pressure Differential } & \text { EASS } & \text { Elliptical/Annular Swept Sector } \\ r & \text { Radial Coordinate } & \text { HSR } & \text { High Speed Research (Program) } \\ x & \text { Streamwise Coordinate } & \text { SSBD } & \text { Shaped Sonic Boom Demonstrator }\end{aligned}$

* Research Scientist, NASA Langley Research Center, Hampton VA 23681

† Aerospace Engineer, NASA Ames Research Center, Moffett Field, CA 94035

‡ Programmer/Analyst, Raytheon Information Solutions, Moffett Field, CA 94035

$\S$ Post-Doctoral Associate, Stanford University, Stanford, CA 94305 


\section{Introduction}

\section{A. Background and Motivation}

The sonic-boom noise levels of today's supersonic aircraft are deemed unacceptable for civil overland flight within the United States [1]. Clearly, the design of a viable supersonic civil transport must involve the reduction of the aircraft's sonic-boom signature to an acceptable level. Dating back four decades and more, members of the research community (e.g., [2-6]) suggested that certain types of vehicle shaping could alter the waveform of an aircraft's ground signature, resulting in a significantly reduced overpressure, relative to that of the familiar N-wave.

The configuration approach to sonic-boom reduction was eventually validated with a flight test [7] in August 2003, during the final phase of the Shaped Sonic Boom Demonstrator (SSBD) Project, jointly conducted by DARPA and NASA at Edwards Air force Base. In this test, a modified F-5E aircraft was shown to generate a "flat-top" shock-wave profile that persisted through the atmosphere to the ground. The demonstration of both the generation and persistence of the F-5E's shaped sonic boom required measurements, not only on the ground track [8], but also near the aircraft in flight [9]. These near-field measurements confirmed that the F-5E's signature had attained its basic wave-form within a relatively small distance from the aircraft. In addition, the wave-form of this near-field signature was predicted, within acceptable accuracy, using computational fluid dynamics (CFD) codes [9].

Now that the potential for "low-boom" aircraft design has been clearly demonstrated, NASA has entered a new phase in sonic boom research. Critical to the development of such designs is the ability to accurately model the generation and propagation of an aircraft's sonic-boom signature. Therefore, an assessment of NASA's current capabilities in sonic-boom prediction is essential to formulating the next steps in testing and tool development within the Supersonics Project. The framework and objectives for this assessment are defined in the following subsection. Note that the present work represents an abridged version of a more comprehensive report [10].

\section{B. Assessment Task Description}

The Supersonics Project has tasked the Cruise Efficiency-Airframe Group to assess NASA's current capabilities for the prediction of near-field sonic boom associated with supersonic aircraft. Any prediction methodology ultimately boils down to the process of modeling, and the most important development challenges faced during the current effort are involved with computational modeling. In particular, it is necessary to discern those traits of a flow solver and a computational mesh that are most beneficial to near-field sonic boom simulation.

From a qualitative perspective, the gridding requirements characterize an accurate near-field sonic boom simulation have been previously determined. That is, in addition to resolving the aircraft geometry and near-field aerodynamics that generate the sonic boom signature, a computational mesh must provide sufficient resolution near shocks to retain steep profiles away from the aircraft. The features in a flow solver that are best suited for sonic boom prediction are also related to the computational mesh, but will require additional discussion, which ensues in the following two subsections.

The major thrust of the present effort is directed toward the use of unstructured grid techniques, because of their efficient implementation for a given model, and their flexibility in adapting to design variation or physical flow features.

The framework and objectives for this assessment task are defined in the following.

NASA's capability to predict near-field sonic boom is judged within the following boundaries:

i. The desired sonic boom prediction is presumed to simulate an aircraft in supersonic cruise. Such advanced topics as aircraft acceleration and maneuvering will be addressed in a later research phase.

ii. Consideration is given to CFD codes and experimental databases that are currently in use by members of the Supersonics Project's Cruise Efficiency-Airframe group at NASA. Neither was additional software developed, nor were tests conducted for this assessment.

The objectives of this assessment task are:

i. Determine what is required of a CFD modeling procedure to adequately predict sonic boom, with respect to the accuracy of the near-field pressure signature as compared to experiment, and the perceived efficiency to support an aircraft design cycle. 
ii. Identify any gaps in the current experimental database that would impact the validation of present and/or future prediction capabilities.

iii. Report findings to the Fundamental Aeronautics Program and make recommendations for the Supersonic Project's next steps in testing (flight and wind tunnel) and tool development.

\section{Sonic Boom Prediction: A Brief Overview}

The prediction of a sonic boom signature on the ground, from an aircraft at high altitude, requires consideration of three flow regions, as sketched in Figure 1. These three regions, referred to simply as the "near," "mid," and "far fields," are bound streamwise by the bow and tail shocks. Although the vertical extent of each flow region is difficult to define, the regions themselves can be characterized with respect to their aerodynamic structure.

The near field is highly nonlinear, with strong streamwise gradients in velocity and pressure, as well as shocks emanating from the aircraft surface. This near field typically extends only a few body lengths from the aircraft. The midfield, usually many times larger than the near field, is the transition region between the near and far fields, and does retain flow features that are related to the aircraft's geometry. Ordinarily, the most extensive of the three regions is the far field, within which the signature's waveform reaches its asymptotic state, most commonly that of an $\mathrm{N}$-wave, as illustrated in Figure 1.

Once the pressure disturbance generated by a supersonic aircraft has reached the far field, it propagates to the ground in a manner that is well predicted by established theory. This theory, initiated by Whitham [11], is incorporated into all of NASA's sonic boom codes [12-14], which can be viewed as propagation codes, as they require input that describes the generation of the shock structure near the aircraft. Conceptually, the input to such a propagation code can be extracted from a CFD calculation. However, the straightforward implementation of this approach involves the issue of "minimum separation distance." That is, one must determine how far from the aircraft the nonlinear near-field flow must be simulated so that the outer boundary of the CFD calculation satisfies the theoretical assumptions inherent in the propagation code. At present, there is no general method by which this separation distance can be predetermined.

\section{Sonic Boom Prediction Methodology: Characterization and Tool Selection}

Although an aircraft's sonic boom, all the way to the ground, could be entirely predicted by a nonlinear, compressible flow code, calculations of such magnitude are currently impractical. Therefore, at present, sonic boom prediction is generally perceived as a two-part procedure: the supersonic flow about an aircraft is modeled by CFD, thereby providing input to a sonic boom propagation code that predicts the signature on the ground. However, the theory of sonic boom propagation formally requires input that is cylindrical in nature, i.e. that the decay of the pressure signature is proportional to $1 / \sqrt{r}$, where $r$ is the radial distance from the aircraft. Referring back to Figure 1, this cylindrical decay is a defining property of the pressure signature in its asymptotic state, which does not occur until propagation in the far field.

As previously stated, this sonic boom theory lies at the heart of all current sonic boom codes, making all of them fundamentally similar in regard to signature propagation. However, the Thomas code [13] was developed to accept a near-field pressure distribution (measured or computed) as input, rather than relying on an "F-function," as originally prescribed by theory [11]. Therefore, the Thomas code is a natural selection for the far-field component of a sonic boom prediction methodology.

Of far greater importance to the present assessment task is concern for the spatial extent of the near-field flow simulation. Any flow code relied upon for this simulation must possess the ability to efficiently calculate the pressure signature well beyond the near field (ideally, to the outer bound of the midfield). By this criterion, there are currently two NASA flow codes that are candidates for incorporation into the desired methodology: FUN3D and CART3D. The distinguishing feature of these analysis codes that supports the criterion is their implementation of an adjoint, output-based adaptation technique (Section II).

In Section II, the output-based grid adaptation technique in FUN3D is discussed, along with supporting test cases. Although CART3D is not discussed in the same regard as FUN3D in the present report, CART3D was an integral part of the parallel research to establish computational grid criteria for near-field sonic boom simulations. 
CART3D and AIRPLANE results, from the parallel study, are presented in Sections III and IV. The final section contains a summary of findings and recommendations for future research.

\section{FUN3D and Adjoint-Based Grid Adaptation Methods}

\section{A. Background}

Solution adaptive grid methodologies have been developed and applied to reduce the required expertise of the practitioner to obtain accurate results or to improve an existing solution. The idea is to increase grid resolution where local discretization error estimates are large, in order to diminish and equally redistribute these estimated errors. A direct approach to such grid adaptation is to identify the appropriate errors by the local magnitudes of equation residuals, solution derivatives, or entropy generation.

However, such local error estimates for the solution-adaptive process tend to focus on the strong features that are present in the flow (e.g., shocks). The manual specification and local-error adaptation methods can miss the connection between the impact of local errors on global output quantities (i.e., lift, drag, sonic boom), and how these local errors are transported through the solution to potentially pollute the results of the calculation.

A potential weakness of local-error adaptation is the under-resolution of important smooth regions of the flow (i.e., supersonic expansions), because such regions do not typically produce strong local-error indicators. Uniformly reducing the errors associated with all local-error sources of the flow may not be optimal from an engineering standpoint, where calculating an output functional (i.e., sonic boom) may be of greater concern. An alternative method is to estimate the error in the calculation of a specified engineering output functional. Output error indicators utilize the dual, or adjoint, problem solution of an output functional to account for the impact of local error, as well as the transport of these local errors throughout the problem domain to improve the calculation of that output functional.

This adjoint-based output adaptation technique has been implemented in two of NASA's large scale CFD codes: FUN3D [15] and CART3D [16], both of which initially implemented the adjoint method for design [17, 18]. During later development [19-21], output-based adaptation was incorporated into both codes, following the method of Venditti and Darmofal $[22,23]$. More details on the output-based method and its development history are available in the introductory sections of References [19-23].

The adaptive FUN3D framework has been applied to sonic-boom problems [24, 25]. These include a generic wing-body configuration designed for "low boom" and multiple cone-cylinder configurations. This earlier work was applied to a wind tunnel test case [26, 27] involving a Mach 2.0 flow over a wing-body geometry referred to as the "Straight-Line Segmented Leading Edge" (SLSLE) model (Figure 2). Although Euler solutions were readily produced (Figure 3), robustness issues were uncovered in the adaptive scheme, and have since been addressed. These robustness issues are associated with grid adaptation, shock capturing, and supersonic expansions.

\section{B. Tetrahedral Cut Cell Method for Local Mesh Refinement}

The three-dimensional propagation of a shock over large distances can introduce anisotropic length scales. Therefore, the flow field must be well resolved in the direction normal to the shock. Furthermore, because the flow field changes slowly in directions tangent to the shock, the demand for computational efficiency would impose a much coarser resolution in those directions. However, the robust production of an arbitrarily adapted, anisotropic body-fitted tetrahedral grid on curved surface is a difficult problem. A "tetrahedral cut cell" approach has been implemented to permit the adaptation of arbitrarily high-aspect ratio tetrahedral grids without the constraint of producing a boundary conforming grid. This method incorporates the ideas of CART3D component-based geometry and the Cartesian cut cell method [28]. A background tetrahedral grid is produced that passes through the body. The volume enclosed by a well resolved triangular surface grid is subtracted from the background grid to obtain the flow domain. This tetrahedral cut-cell method was used to simulate a sonic boom signature at 10 body lengths away from the SLSLE wing-body model, as displayed in Figure 4.

The discrete adjoint system of equations can be solved with an iterative scheme that is, itself, discretely adjoint to the primal solution scheme. The benefit of such an algorithm is that the nonlinear, linearized-forward, and adjoint systems are all guaranteed to converge at the same asymptotic rate [29, 30]. However, in the event that the linearized forward system of equations becomes unstable, the adjoint solution will also become unstable, because 
the asymptotic rates of convergence for the two systems are ultimately determined by the same set of eigenvalues. It has been observed in practice that the use of non-differentiable reconstruction limiters can admit such problems and compromise the robustness of the overall adaptive procedure on the finer mesh levels. Instead, in a manner similar to that in Reference [31], a continuously-differentiable, heuristic pressure-based limiter used to improve the stability of the linearized system of equations for both the forward and reverse integrations.

Without the viscosity that is present in physical flow, the Euler equations can permit rapid expansions around sharp corners in supersonic flow. These sharp corners appear at thin wing leading edges and the backward facing steps of finite-thickness trailing edges. The dissipation that is present in numerical schemes can mimic viscosity and prevent the potentially catastrophic expansions from reaching unrealizable states. However, adaptation schemes are designed to reduce this numerical dissipation to produce more accurate results, which exacerbates the problem. Therefore, artificial viscosity [32] is added to the Euler equations in order to control these expansions. In addition, a transpiration boundary condition is applied at blunt trailing edges to reduce expansion strengths in those locations.

\section{Computational Mesh Criteria}

\section{A. Simulation Tools}

The two aerodynamic flow codes discussed in this section are AIRPLANE and CART3D. Both are high-fidelity analysis codes that solve the three-dimensional Euler equations on unstructured grids, and can be applied to complex geometries with adaptively refined meshes. The AIRPLANE code, designed for tetrahedral meshes, originated during the late 1980's [15-35], and has remained in continuous development. Tetrahedral grids for AIRPLANE are produced with MESH3D [36-38], which automatically generates tetrahedra in the flow volume for a given surface triangulation. CART3D [16] is a Cartesian-grid based flow code that is bundled within an automated package that includes mesh generation.

The accuracy of this CFD approach to sonic boom prediction is presently determined by validation with experimental measurement. To compare the simulated sonic boom signatures with wind tunnel data, the near-field CFD is extrapolated to a specified distance from the model, to match the measurement location. This extrapolation is accomplished by taking the near-field CFD solution at some distance $h$ from the model surface, and then using it as input to ANET, a subroutine that implements the sonic boom propagation method developed by Thomas [13].

\section{B. Background and Objectives}

During the earlier phase of the High Speed Research (HSR) Program, AIRPLANE was employed to evaluate and design sonic boom configurations for several aircraft models (e.g., [39]). Sonic boom signatures were extrapolated to the mid and far field by inputting the AIRPLANE calculation at distances of $h / L=0.1$ to $h / L=0.33$ from the model surface, where $L$ is a characteristic length of the model. MESH3D was subsequently developed to support the aerodynamic performance phase of the HSR program. As a result, its grid generation properties were not ideal for sonic boom computations, most significantly the rapid mesh coarsening away from the body surface. Therefore, this initial study has been focused, in large part, on the enhancement of MESH3D to support sonic boom calculations, which must include high grid resolution in the vicinity of shocks, while retaining sufficient support for the near-field aerodynamics, and without sacrificing computational efficiency.

The technical objectives of the present study are tied to the desire to retain the computational efficiency previously established for these simulation tools in regard to aerodynamic design optimization. As such, accurately simulated pressure signatures are extrapolated from $h / L=0.4$, only a slightly greater separation distance than was obtained during the early HSR work. This approach allows for a more test cases to be run within the allotted schedule. In addition, the volume grid density must be sufficient high within the sonic boom region of influence (between the bow and tail shocks) so that alterations in grid density are not required for variations in configuration.

During previous research, MESH3D was modified to permit the user to specify one or more refinement boxes where the edges of the tetrahedra are split. At the same time, spherical refinement regions were also included. Use of the spherical and rectangular refinement regions enabled sonic boom calculations to be performed, but they were not ideal and required more grid points than necessary, as will become clear in the first test case below. 


\section{Mesh Refinement Strategy: Elliptical/Annular Swept Sectors}

\section{AIRPLANE Methodology}

MESH3D has been more recently modified to include a third type of region refinement strategy, referred to as an Elliptical/Annular Swept Sector (EASS). This approach to localized mesh refinement has been developed specifically for sonic boom computations. The user input parameters are $x_{1}, x_{2}, y_{1}, y_{2}, z_{1}, z_{2}$, the Mach number, and the azimuthal angle, $\phi$. The EASS region can be annular (circular in axial view) when $y_{1}=z_{1}$ and $y_{2}=z_{2}$. An elliptically shaped refinement region is necessary for winged component regions, in order to refine some distance from the surface when the grid density is insufficient for sonic boom calculations, e.g., outboard of a wing tip. Annular shaped regions are convenient for axisymmetric configurations, or aircraft axisymmetric components within a configuration. An EASS refinement region can be contained beneath the aircraft for small $\phi$, or the refinement region can extend to the upper symmetry plane with $\phi>90^{\circ}$. EASS mesh refinement enables efficient and accurate sonic-boom assessment that is compatible with optimization, provided the aircraft bow and tail shocks are contained within appropriate EASS regions.

\section{CART3D Methodology}

When CART3D is applied to a typical aerodynamic problem, a Cartesian mesh coarsens by multiples of two from the surface of the vehicle to the far field boundaries. However, the logical structure within the CART3D flow solver enables independent grid generation processes for the surface geometry and the flow volume. Therefore, users can easily maintain higher grid density away from the surface by volume-grid refinement in pre-specified rectangular regions. Such characteristics are advantageous to sonic boom analysis, in that the effects of overall grid variation can easily be eliminated from a given problem, because the user can independently analyze the effects of grid density on the surface and in the flow field. The EASS refinement strategy has been incorporated into CART3D by way of a Matlab-based code that generates a large number of rectangular regions that essentially fill any EASS refinement regions prescribed by the user input parameters.

\section{Wind Tunnel Test Cases}

Five experimental configurations from References [40-42] have been simulated with AIRPLANE and CART3D, two of which are presented in this abridged report. The selected models, shown with simulated test conditions in Table 1, are those referred to as Models " $\mathrm{B}$ " and " $\mathrm{E}$ " in the full assessment report [10].

\section{Test Case: Model B, Cone-Cylinder, Mach 1.41 and 2.01}

Wind tunnel test cases for a $3.24^{\circ}$ half-angle cone-cylinder (Model B, Table 1) are modeled at two Mach numbers. The wind tunnel models in the original test program [40] were all 2.0 inches in length, and included bodies of revolution of varying leading-edge bluntness. The experimental measurements were obtained in NASA Langley's $4 \times 4$-foot Unitary Wind Tunnel. In addition to evaluating the performance of the CFD modeling process, validating simulations at multiple tunnel speeds can be helpful in determining whether the prediction accuracy is sensitive to Mach number.

Various refinement regions were evaluated in an attempt to accurately compute the sonic-boom pressure signature of the cone-cylinder model. Included in the process were rectangular and spherical regions, which were already implemented from previous HSR work. The rectangular and spherical refinement regions were inefficient when relatively few regions were required, producing meshes with more than four times the total number of grid points than the eventual EASS region. A larger number of rectangular meshes could have been used to fill an EASS region (as is done with Cart3D); however, that strategy was previously attempted during the HSR program [39], and the EASS refinement approach was developed to replace it.

In Figure 6, AIRPLANE and CART3D simulations are extrapolated from $h / L=0.4$ to $h / L=10$, and compared with experimental measurement, for $M=1.41$ and 2.01. The agreement with experiment is noticeably better at Mach 1.41 than at Mach 2.01, suggesting that the prediction process is sensitive to Mach number. Also, note that various differences in the refinement region had little effect on the near-field signatures. Although slight changes are observed in the expansion region for the largest mesh, the number of grid cells involved in this case would be impractical for a design optimization environment. 


\section{Test Case: Model E, Delta Wing-Body, Mach 1.68}

The delta wing-body configuration, analyzed with AIRPLANE and CART3D, is from Ref. [42]. In the surface geometry in Figure 7, note that the sting/model junction, which ordinarily involves a blunt insertion into the model cavity, is instead configured with a 12-degree sloped truncated cone.

The EASS refinement regions for the AIRPLANE calculations are shown superimposed in Figure 8. Three sets of two refinements were needed (sets are distinguished by color). The splitting of the tetrahedral edges requires multiple regions to maintain a desired level of refinement throughout the region. The delta-wing EASS refinement regions were obtained by studying the moderate lift case $\left(\alpha=2.56^{\circ}\right)$, and then applying the same regions to the non-lifting ( $\alpha=0$ ) and higher lift ( $\alpha=4.74^{\circ}$ ) cases. The bow shock was not adequately resolved until a third set of refinement regions was applied. This third set is represented by the red regions in Figure 8.

The pressure signatures for the EASS study for $M=1.68$ and $\alpha=2.56^{\circ}$, are shown in Figure 8. The bow and wing shocks show excellent agreement with experiment. Note the "additional shock" due to the truncated cone modeled in the geometry between the model base and the sting. The non-lifting case is shown in Figure 10. The strength of the wing shock is slightly under predicted by both AIRPLANE and CART3D. The higher lift case is shown in Figure 11, and the agreement with experiment is very good. There are slight over-predictions in the bow and wing shocks from both CFD methods. These minor overshoots may be due to a slight over-prediction of $\alpha$, as it was determined by an inviscid flow calculation with the experimentally measured $C_{L}$ as input. Note that, in this case, the 12-degree-sloped collar at the base/sting junction shows up as a small blip in the expansion region between the wing and tail shocks.

\section{Surface Geometry Criteria}

\section{A. Simulation Tools}

The sonic boom predictions discussed in this section were performed with the AIRPLANE code, using MESH3D for grid generation, and ANET for signature extrapolation, all of which were previously described. The delta wing-body from the previous section was evaluated to assess the effects of surface grid refinement on sonic boom predictions. The objective for this exercise is to determine whether near-field sonic boom calculations require highly refined surface definitions, as this situation would severely impact the overall grid size for mesh generation schemes (such as MESH3D) in which a surface triangulation is topologically connected to the volume grid. Therefore, CART3D was not evaluated during this particular research phase, because CART3D's logical structure allows surface and volume meshes to be refined independently.

\section{B. Wind Tunnel Test Case: Delta Wing-Body}

For the delta wing-body test case, AIRPLANE solutions on two surface grids are compared. This configuration was originally defined analytically, so a special purpose program was written to generate both surface meshes. The surface grids near the nose of the forebody are shown in Figure 10a. The meshes at the wing body junction, in the vicinity of the leading edge, are shown in Figure 10b. The surface meshes consist of 21,018 points for the original mesh (red grid in the lower halves), and 45,025 points in the fine mesh (green grid in the upper halves).

The symmetry plane mesh for the AIRPLANE solution, colored to represent the $C_{P}$ distribution, is shown in Figure 13. The coarser surface grid was used for the computations in this refinement study. Each surface triangle is a tetrahedral face in the volume grid, so the volume mesh near the surface would be approximately twice as dense if the finer surface mesh had been used.

Refinement regions are now prescribed the EASS strategy described in Section II. Figure 13 shows all attempts, along with the number of total grid points, to indicate the amount of refinement needed. The fifth attempt, EASS-5, is judged to match well with experiment. The EASS-5 refinement region was applied to the newly refined surface grid, and there is no significant difference between the two solutions, as the brown curve lies nearly directly over the cyan curve. In spite of these and other efforts, highly refined surface grids did not increase the accuracy of the sonic boom computations. However, this is a desirable outcome, as it indicates that specialized and/or highly refined surface grids are not an issue for accurate near-field sonic boom predictions. 


\section{Summary of Findings and Recommendations}

The Supersonics Project has embarked upon the extension of NASA's current simulation tools to sonic boom prediction. The present report represents an abridged version of a comprehensive assessment [10] of current capabilities in near-field sonic boom prediction for the purpose of formulating the next steps in testing and tool development. The focus of the present report is on the effectiveness of three CFD codes: AIRPLANE, CART3D, and FUN3D. All three codes have been enhanced with new mesh refinement strategies that were developed specifically for sonic boom calculations. Research level applications for these new approaches involved the modeling of wind tunnel tests and comparison to experimental measurement. The following list contains the major findings resulting from the research reported herein, including any corresponding recommendations.

i. An anisotropic mesh refinement approach was demonstrated with a FUN3D simulation in which a sonic boom signature was accurately predicted, by direct computation, out to a separation distance of 10 body lengths, i.e., well into the midfield flow. It is conceivable that such a capability, if applied over time to many configurations, could provide valuable benchmark data to foster an empirical method by which a reasonable approximation for a minimum separation distance could be determined before running a simulation on a given aircraft configuration.

ii. Theoretically extrapolated signatures taken from near-field AIRPLANE and CART3D calculations compared well with wind tunnel validation data. The EASS strategy has proved effective, without regard for a specialized and/or highly refined surface grid,

iii. Near-field sonic boom predictions for a delta wing-body were found to be virtually identical when the geometry was defined by a surface mesh of either medium or fine resolution (Figure 13). Although the surface-grid refinement study in section III is admittedly limited, the result suggests that surface mesh criteria for acceptable near-field sonic boom simulation are similar to those for the accurate prediction of aerodynamic forces and moments.

iv. The cone-cylinder results in Figure 6 (as well as those for two other models in Reference [10]) suggest that the accuracy for near-field sonic boom prediction could degrade with increasing Mach number. However, this issue most likely arises from the weak-shock theory embedded within the propagation method used to extrapolate the CFD solution to the midfield. Testing this assumption would require comparison of the extrapolated signatures to signatures that are computed without extrapolation, on a mesh that is sufficiently refined out to the distance at which the wind tunnel flow was probed.

$v$. Given the encouraging nature of the preliminary results presented herein, it is reasonable to expect the expeditious development of an efficient sonic boom prediction methodology that will eventually become compatible with an optimization environment.

\section{References}

1. Civil Aircraft Sonic Boom, Section 91.817 in Code of Federal Regulations, Title 14.

2. Jones, L. B., “Lower Bounds for Sonic Bangs,” Journal of the Royal Aeronautical Society, Vol. 65, June 1961, pp. 433-436.

3. Carlson, H. W., "The Lower Bound of Attainable Sonic-Boom Overpressure and Design Methods of Approaching this Limit,” NASA TN-D-1494, Oct. 1962.

4. McLean, F. E. and Shrout, B. L., "Design Methods for Minimization of Sonic Boom Pressure-Field Disturbances,” Journal of the Acoustical Society of America, Vol. 39, No. 5, Part 2, 1966, pp. S19-S25.

5. George, A. R., "Lower Bounds for Sonic Booms in the Mid-Field,” AIAA Journal, Vol. 7, No. 8, Aug. 1969, pp. $1542-1545$.

6. George, A. R. and Seebass, R., “Sonic Boom Minimization Including Both Front and Rear Shocks,” AIAA Journal, Vol. 9, No. 10, Oct. 1971, pp. 2091-2093.

7. Pawlowski, J. W., Graham, D. H., Boccadoro, C. H., Coen, P. G., and Maglieri, D. J., “Origins and Overview of the Shaped Sonic Boom Demonstration Program,” AIAA-2005-0005, 2005.

8. Plotkin, K. J., Haering, E. A., Murray, J. E., Maglieri, D. J., Salamone, J., Sullivan, B. M., and Schein, D., “Ground Data Collection of Shaped Sonic Boom Experiment Aircraft Pressure Signatures,” AIAA-2005-0010, 2005.

9. Haering, E. A., Jr., Murray, J. E., Purifoy, D. D., Graham, D. H., Meredith, K. B., Ashburn, C. E., and Stucky, M., Lt. Col., "Airborne Shaped Sonic Boom Demonstration Pressure Measurements with Computational Fluid Dynamics Comparisons," AIAA-2005-0009, 2005. 
10. Bangert, L. S., Campbell, R. L., Carter, M. B., Casper, J. H., Cliff, S. E., Coen, P. G., Deere, K. A., Durston, D. A., Haering, E. A., Melton, J. E., Moes, T. R., Nielsen, E. J., Park, M. A., Wood, R. M., Bobbitt, P. J., Maglieri, D. J., McMullen, M. S., and Thomas, S. D., "State of the Art Assessment for Near-Field Sonic Boom and Performance Prediction," NASA Technical Memorandum, 2008 (in preparation).

11. Whitham, G. B., “The Flow Pattern of a Supersonic Projectile,” Communications on Pure and Applied Mathematics, Vol. 5, 1952, pp. 301-348.

12. Hayes, W. D., Haefeli, R. C. and Kulsrud, H. E. "Sonic Boom Propagation in a Stratified Atmosphere, with Computer Program,” NASA CR-1299, April 1969.

13. Thomas, C.L., "Extrapolation of Sonic Boom Pressure Signatures by the Waveform Parameter Method,” NASA TN-D-6832, June 1972.

14. Plotkin, K.J., “PCBoom3 Sonic Boom Prediction Model, Version 1.0e,” Wyle Research Report WR95-22E, October 1998.

15. “FUN3D Home Page,” http://fun3d.larc.nasa.gov .

16. “CART3D Home Page,” http://people.nas.nasa.gov/ aftosmis/cart3d/ .

17. Nemec, M.N., and Aftosmis, M.J., “Aerodynamic Shape Optimization Using a Cartesian Adjoint Method and CAD Geometry,” AIAA Paper 2006-0345.

18. Nielsen, E. J. and Anderson, W. K, “Recent Improvements in Aerodynamic Design Optimization on Unstructured Meshes,” AIAA Paper 2001-0596.

19. Park, M. A., “Adjoint-Based, Three-Dimensional Error Prediction and Grid Adaptation,” AIAA Journal, Vol. 42, No. 9, 2004, pp. 1854-1862; also AIAA Paper 2002-3286.

20. Park, M. A., “Three-Dimensional Turbulent RANS Adjoint-Based Error Correction,” AIAA Paper 2003-3849, June 2003.

21. Nemec, M. N. and Aftosmis, M. J., “Adjoint Error Estimation and Adaptive Refinement for Embedded-Boundary Cartesian Meshes,” AIAA Paper 2007-4187, Jan. 2007.

22. Venditti, D. A. and Darmofal, D. L., "Grid Adaptation for Functional Outputs: Application to Two-Dimensional Inviscid Flows,” Journal Computational Physics, Vol. 176, 2002, pp. 40-69; also AIAA Paper 2000-2244.

23. Venditti, D. A. and Darmofal, D. L., “Anisotropic Grid Adaptation for Functional Outputs: Application to Two-Dimensional Viscous Flows,” Journal Computational Physics, Vol. 187, 2003, pp. 22-46.

24. Lee-Rausch, E. M., Park, M. A., Jones, W. T., Hammond, D. P., and Nielsen, E. J., “Application of Parallel Adjoint-Based Error Estimation and Anisotropic Grid Adaptation for Three-Dimensional Aerospace Configurations,” AIAA $2005-4842$.

25. Jones, W. T., Nielsen, E. J., and Park, M. A., "Validation of 3D Adjoint Based Error Estimation and Mesh Adaptation for Sonic Boom Prediction,” AIAA-2006-1150, Jan 2006.

26. Mack. R. J. and Kuhn, N., "Determine of Extrapolation Distance with Measured Pressure Signatures from Two Low-Boom Models,” NASA TM-2004-213264, November 2004.

27. Mack. R. J. and Kuhn, N., "Determine of Extrapolation Distance with Pressure Signatures Measured at Two to Twenty Span Lengths from Two Low-Boom Models,” NASA TM- 2006-214524, November 2006.

28. Aftosmis, M. J., Berger, M. J., and Melton, J. E., "Robust and Efficient Cartesian Mesh Generation for Component-Based Geometry,” AIAA Paper 1997-0916.

29. Nielsen, E. J., Lu, J., Park, M. A., and Darmofal, D. L., “An Implicit, Exact Dual Adjoint Solution Method for Turbulent Flows on Unstructured Grids, Computers \& Fluids, Vol. 33, 2004, pp. 1131-1155.

30. Giles, M., Duta, M., Muller, J. D., and Pierce, N., “Algorithm Developments for Discrete Adjoint Methods,” AIAA Journal, Vol. 41, No. 2, 2003, pp. 198-205.

31. Gnoffo, P. A. and White, J. A., “Computational Aerothermodynamic Simulation Issues on Unstructured Grids,” AIAA Paper 2004-2371, 2004.

32. Barter, G. E. and Darmofal, D. L., “Shock-Capturing with Higher-Order, PDE-Based Artificial Viscosity,” AIAA 2007-3823.

33. Jameson, A., Baker, T. J. and Weatherill, N. P., “Calculation of Inviscid Transonic Flow over a Complete Aircraft,” AIAA Paper 1086-0103, January 1986.

34. Jameson, A. and Baker, T. J., "Euler Calculations for a Complete Aircraft," Tenth International Conference on Numerical Methods in Fluid Dynamics, Bejing, China, June 1986.

35. Jameson, A. and Baker, T. J., “Improvements to the Aircraft Euler Method,” AIAA Paper 1987-0452, January 1987.

36. Baker, T. J., “Triangulations, Mesh Generation and Point Placement Strategies,” in Frontiers of Computational Fluid Dynamics, Caughey and Hafez, eds., J. Wiley \& Sons, 1994.

37. Baker, T. J., and Vassberg, J. C., “Tetrahedral Mesh Generation and Optimization,” Proceedings of the 6th International Conference on Numerical Grid Generation, Greenwich, UK, July 1998, pp. 337-349. 
38. Thomas, S. D., Baker, T. J., and Cliff, S. E., "Improvements to the Unstructured Mesh Generator MESH3D,” NASA High Speed Research Program, Aerodynamic Performance Workshop, Part 1, Volume 1, 1999, pp. 213 -252.

39. Cliff, S. E., "Computational/Experimental Analysis of Three Low Sonic Boom Configurations with Design Modifications,” NASA CP-3172, High Speed Research Sonic Boom Workshop Vols. I \& II, February 1992, pp 89-117.

40. Carlson, H. W., Mack, R. J., and Morris, O. A.., “A Wind Tunnel Investigation of the Effect of Body Shape on Sonic Boom Pressure Distributions,” NASA TN-D-3106, November 1965.

41. Mendoza, J. P. and Hicks, R. M., "Further Studies of the Extrapolation of Near Field Overpressure Data,” NASA TM-X2219, March 1971.

42. Hunton, L. W., Mendoza, J. P. and Hicks, R. M., “Some Effects of Wing Planform on Sonic Boom,” NASA TN-D-7160, January 1973.

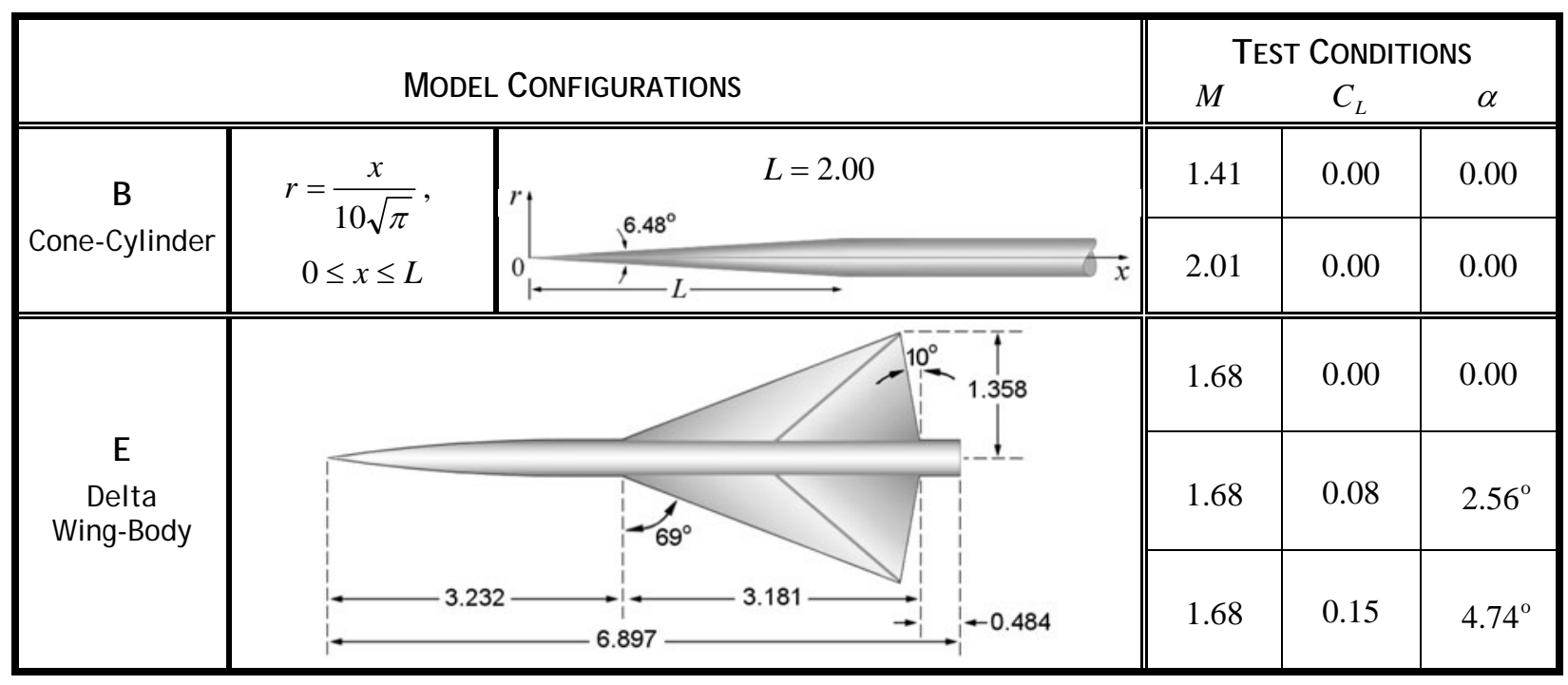

Table 1. Test configurations. Model dimensions in inches. $C_{L}$ measured in all cases. Non-zero $\alpha$ determined by inviscid flow calculation. Model B has a cylindrical afterbody of radius $0.2 L / \sqrt{\pi}$ in.

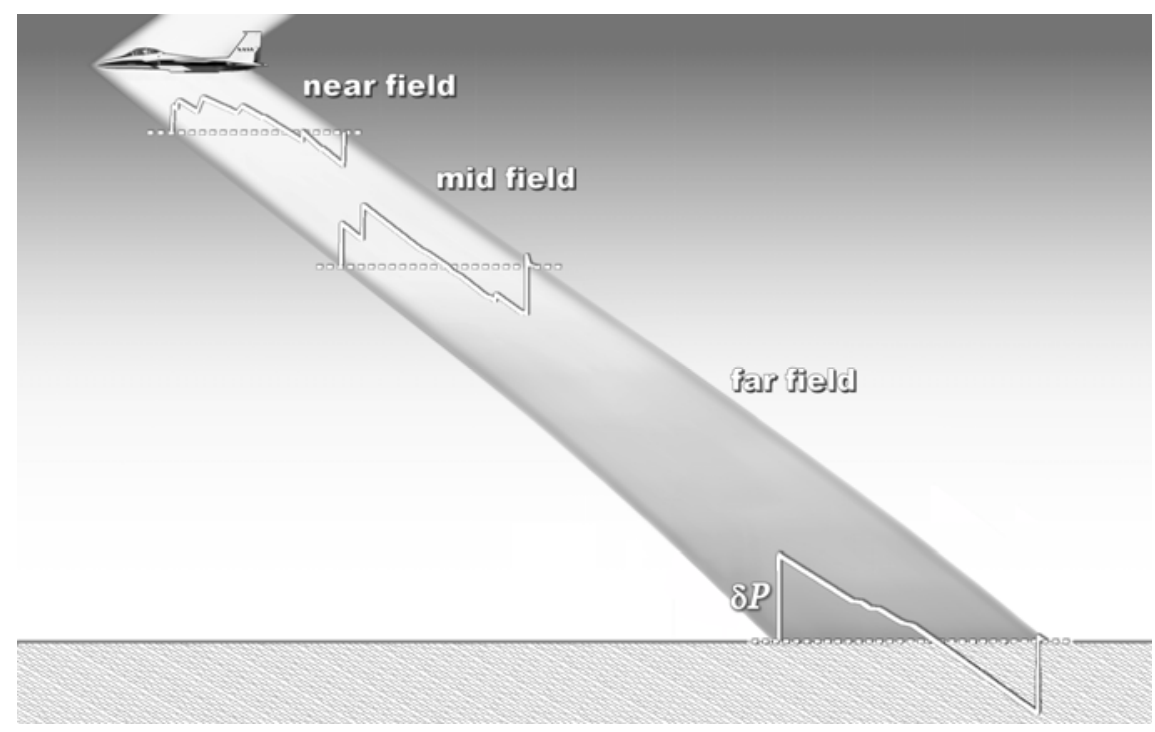

Figure 1. Schematic for sonic boom propagation. 


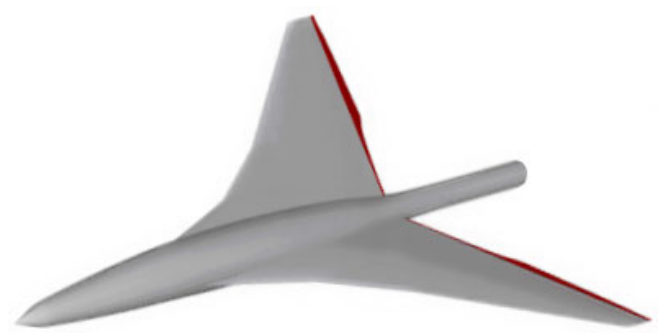

Figure 2. SLSLE model geometry. Red area denotes sharpened trailing edge.

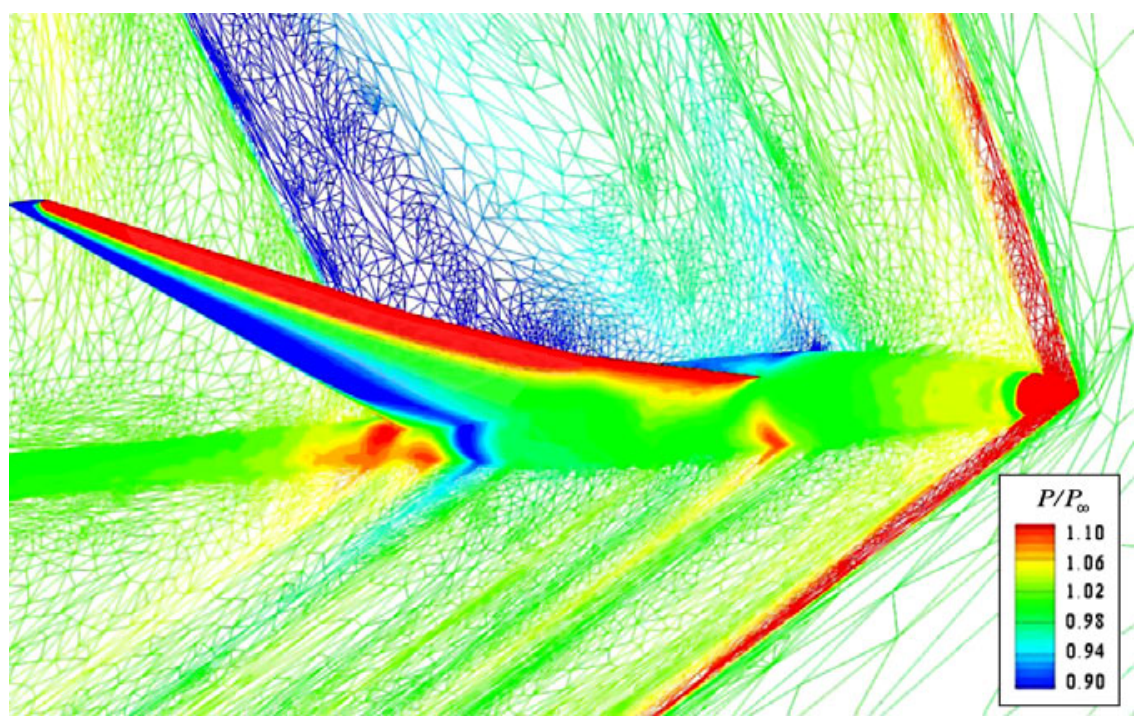

Figure 3. FUN3D Simulation: SLSLE model, symmetry plane, grid and pressure contours, $M=2.0$

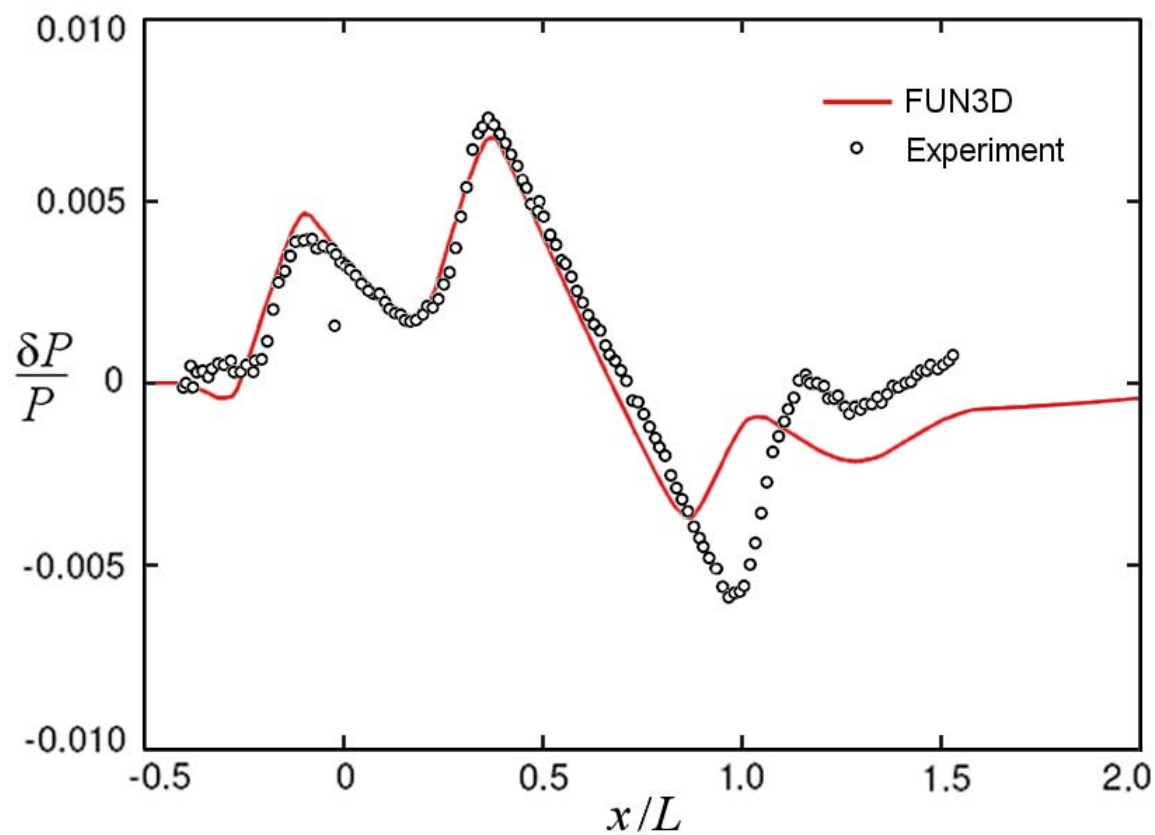

Figure 4. Sonic boom signature, SLSE model, $h / L=10, \phi=30^{\circ}$, Mach 2.0. 


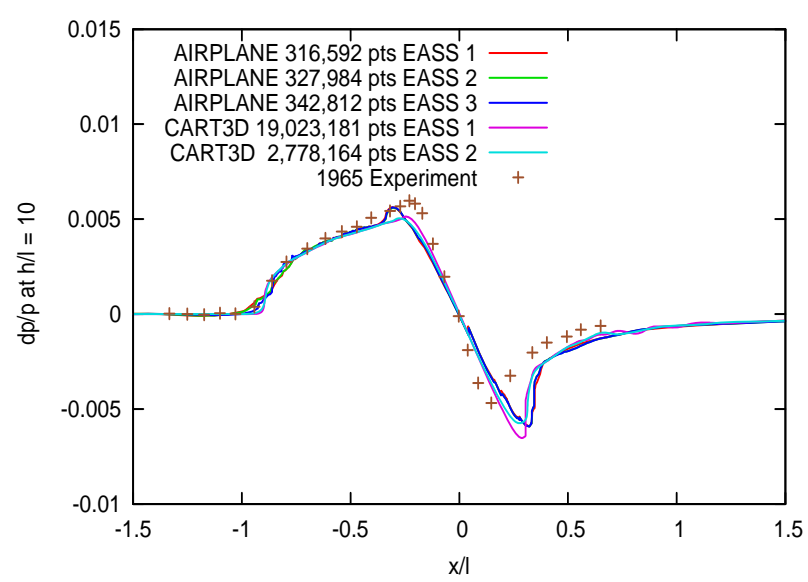

(a) $M=2.01$

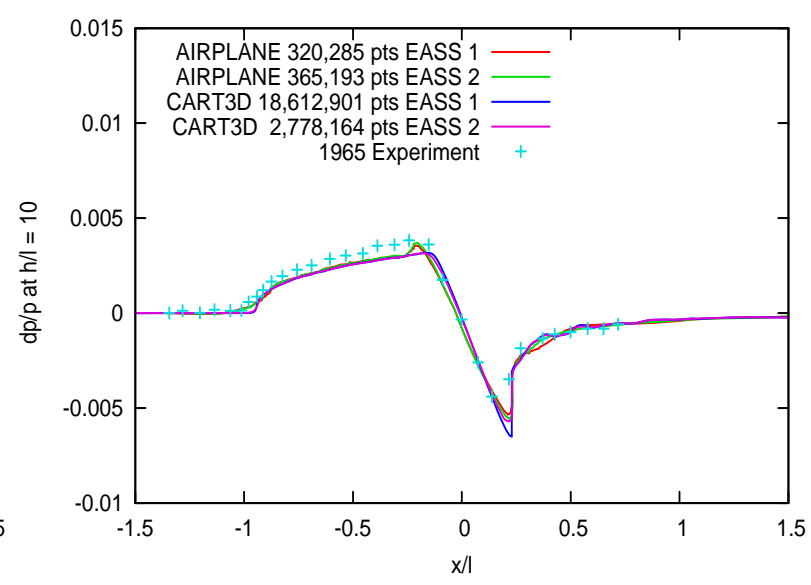

(b) $M=1.41$

Figure 5. Cone-cylinder, pressure signature, $\alpha=0$, CFD data extrapolated from $0.4 \mathrm{~h} / \mathrm{L}$ to $10 \mathrm{~h} / \mathrm{L}$, via ANET, uniform atmosphere.

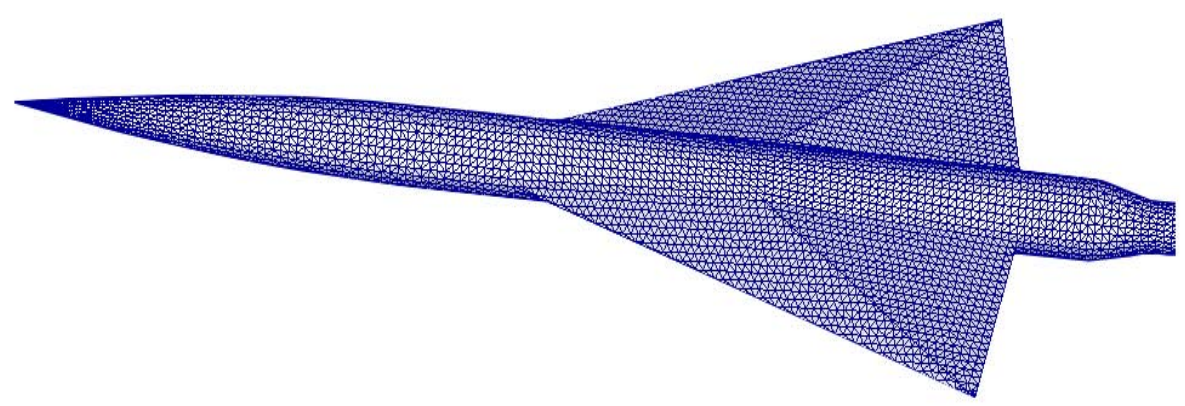

Figure 6. Delta wing-body, surface grid.

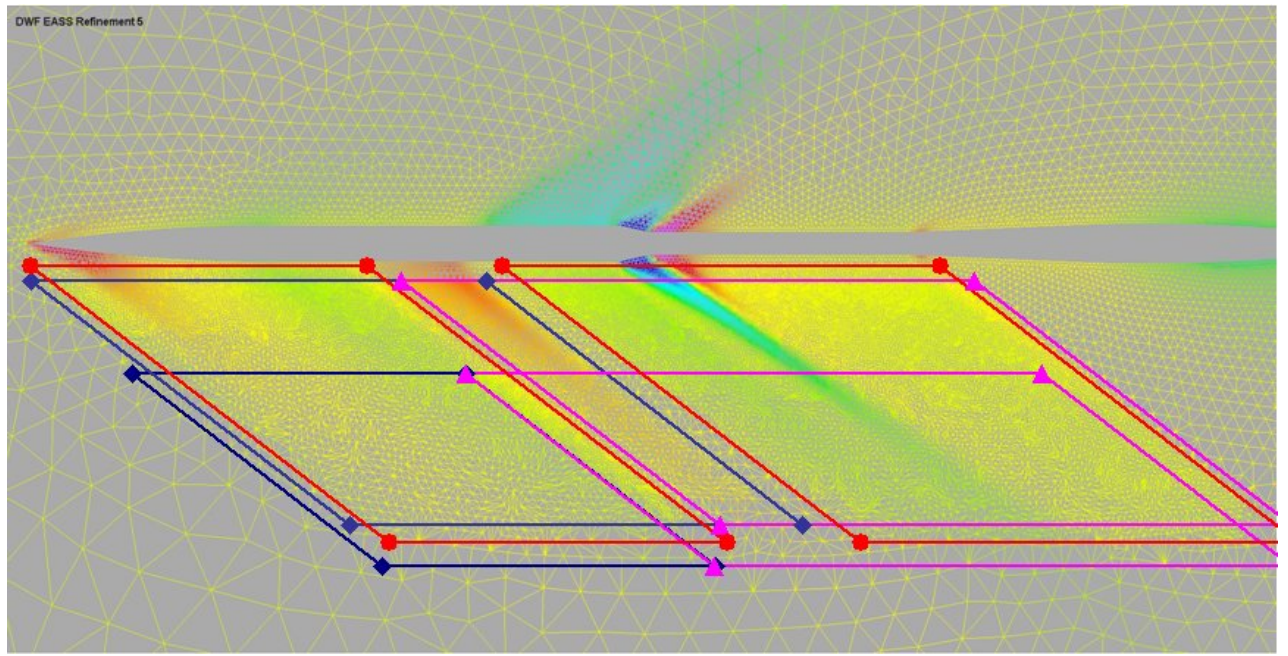

Figure 7. Refinement region boundaries, symmetry plane, Delta Wing-Body (EASS 5), AIRPLANE solution, $M=1.68, \alpha=2.56^{\circ}$. 


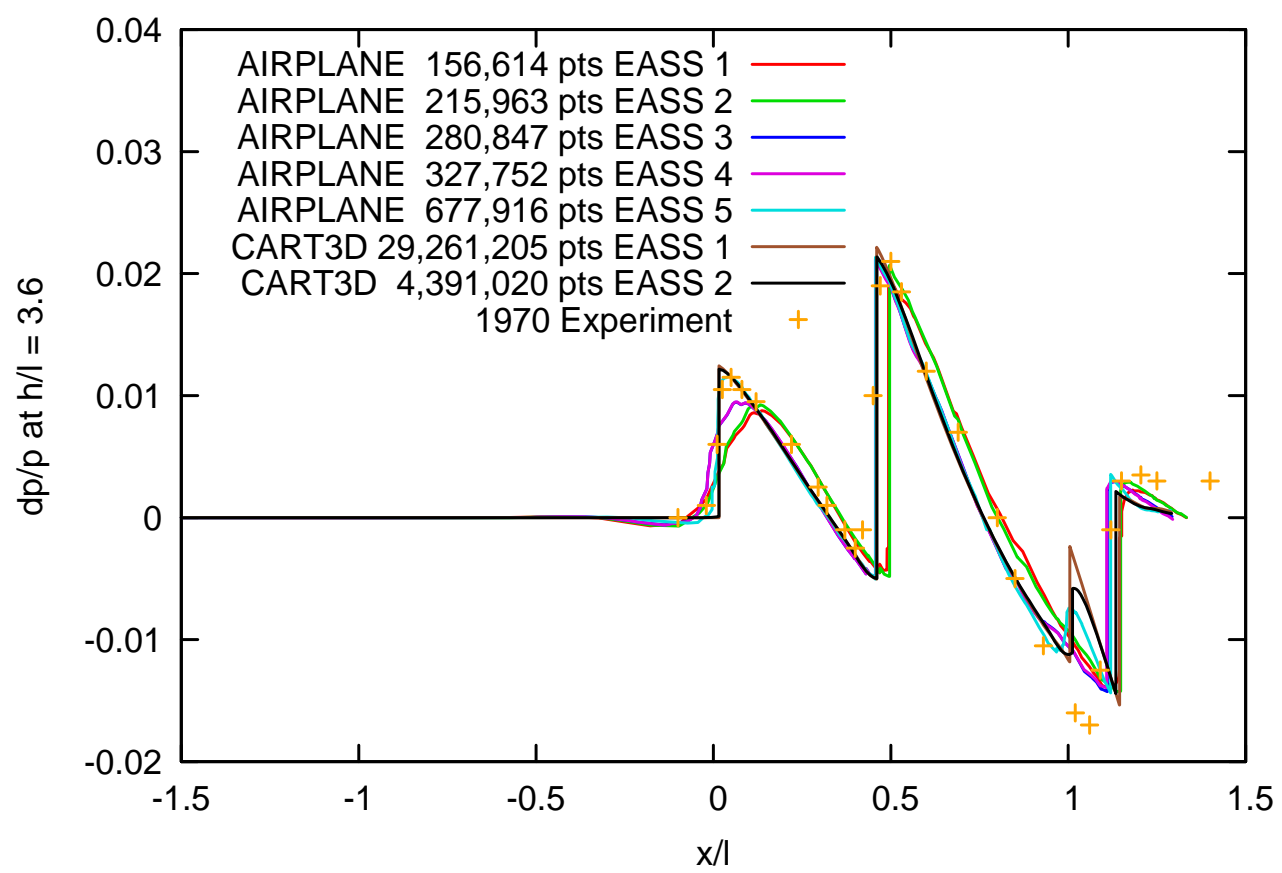

Figure 8. Delta Wing Body, $M=1.68, \alpha=2.56^{\circ}, C_{L}=0.08$, CFD data extrapolated from $0.4 h / L$ to $3.6 h / L$.

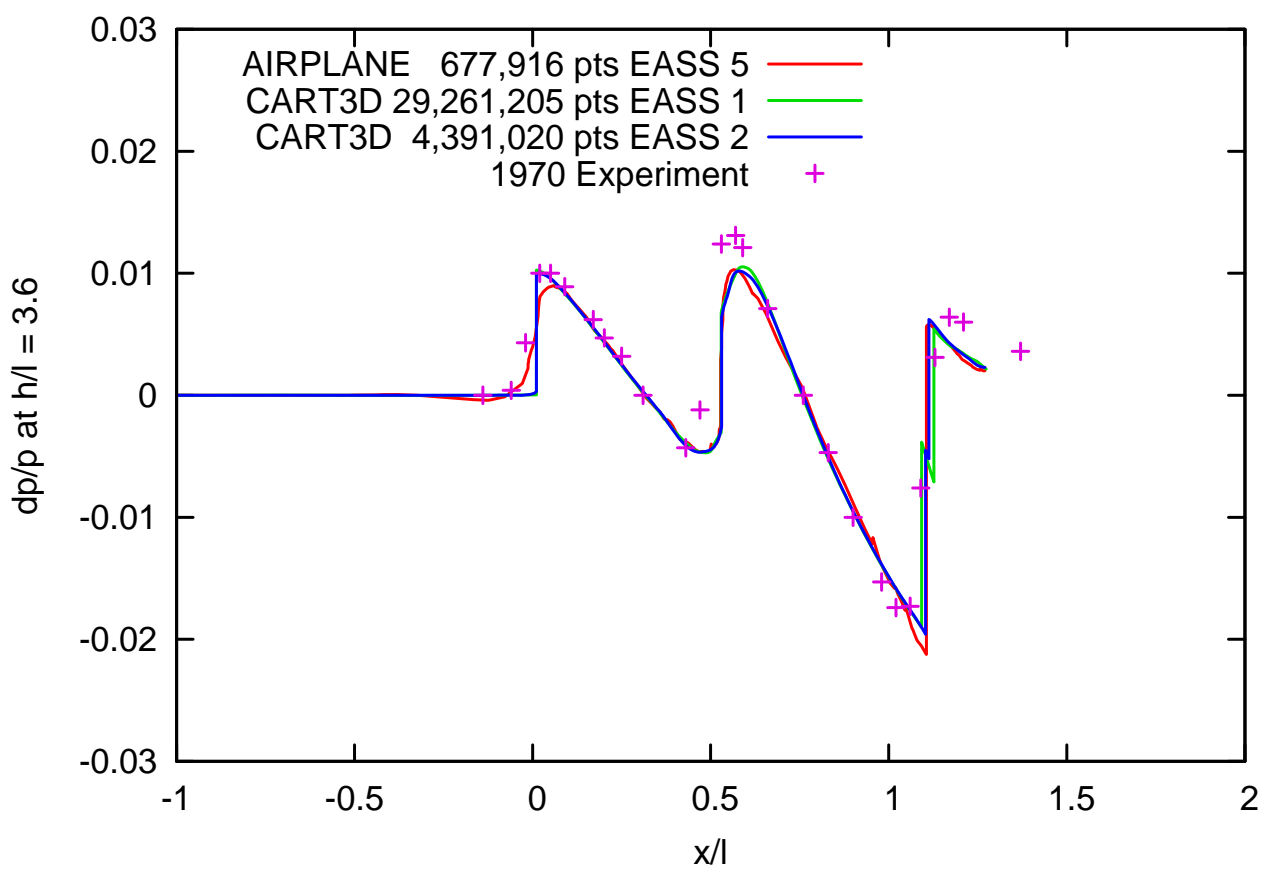

Figure 9. Delta Wing Body, $M=1.68, \alpha=0, C_{L}=0.00$, CFD data extrapolated from $0.4 h / L$ to $3.6 h / L$. 


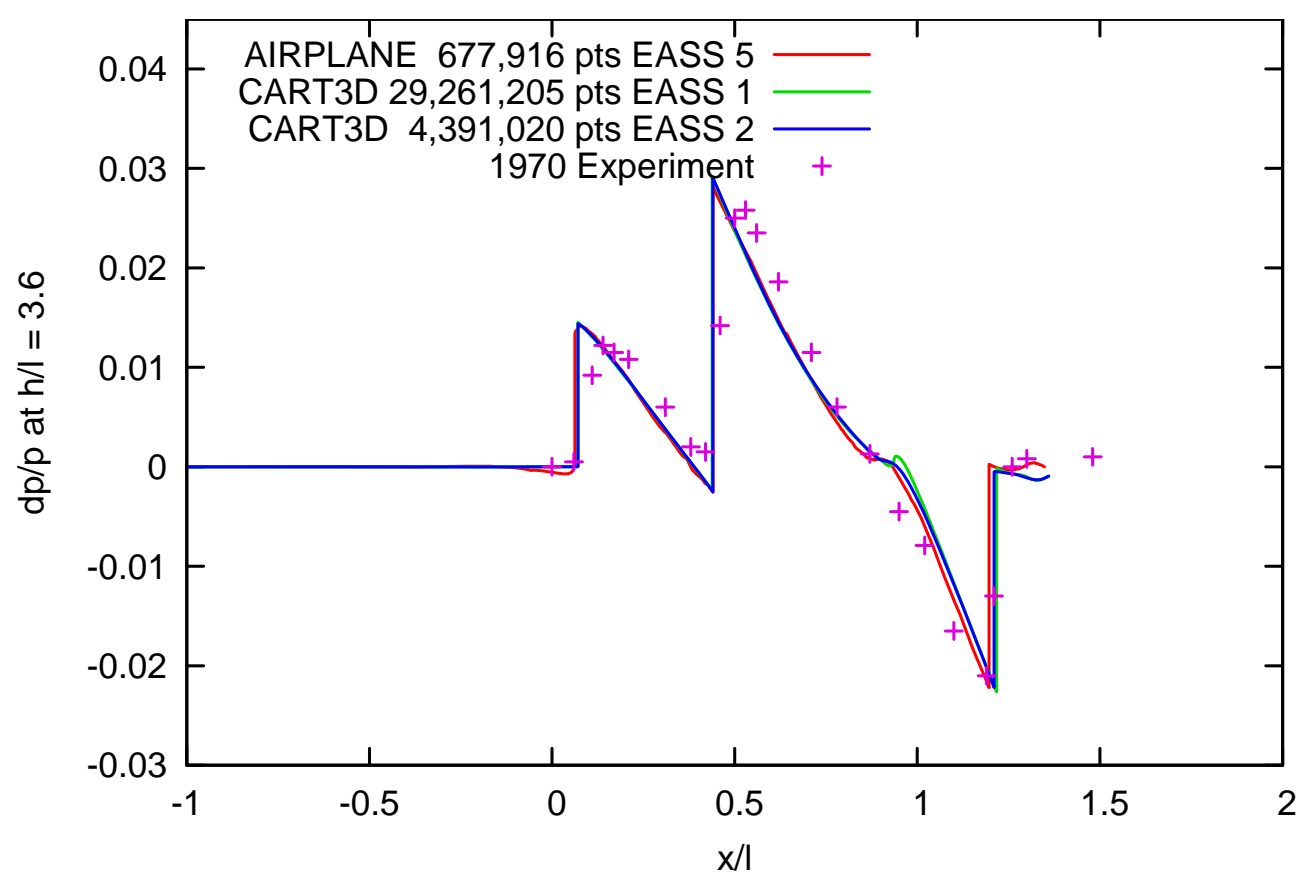

Figure 11. Delta Wing Body, $M=1.68, \alpha=4.74^{\circ}, C_{L}=0.15$, CFD data extrapolated from $0.4 h / L$ to $3.6 h / L$.

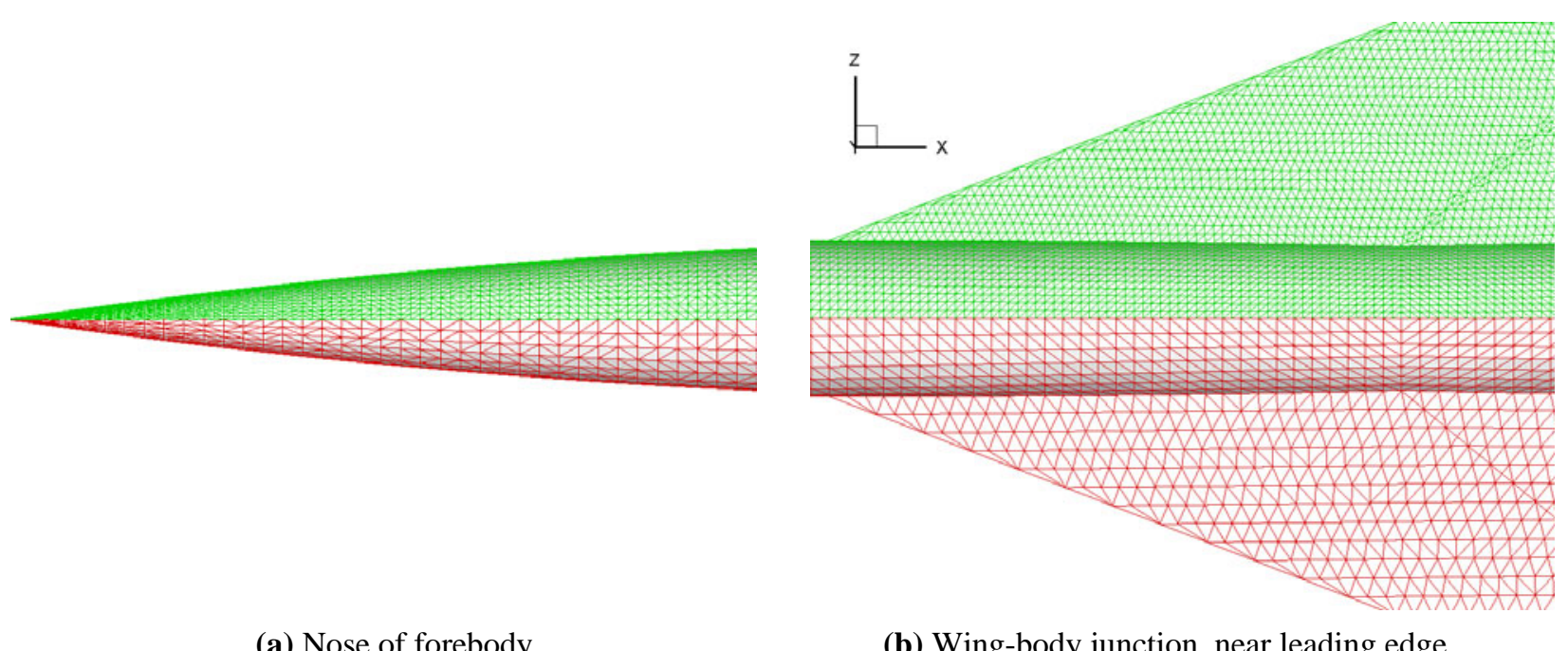

(a) Nose of forebody.

(b) Wing-body junction, near leading edge.

Figure 10. Delta wing-body, surface grids. 


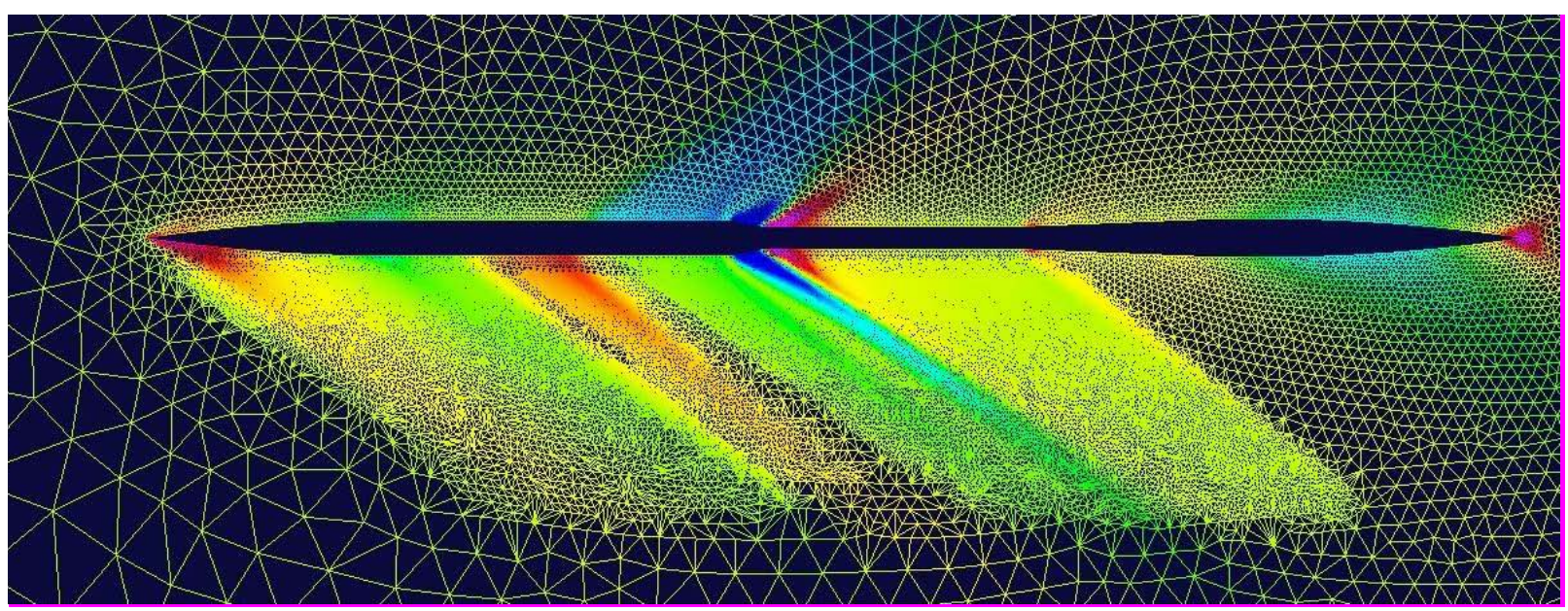

Figure 12. Delta wing-body, symmetry plane mesh. Color contours represent $C_{P}$.

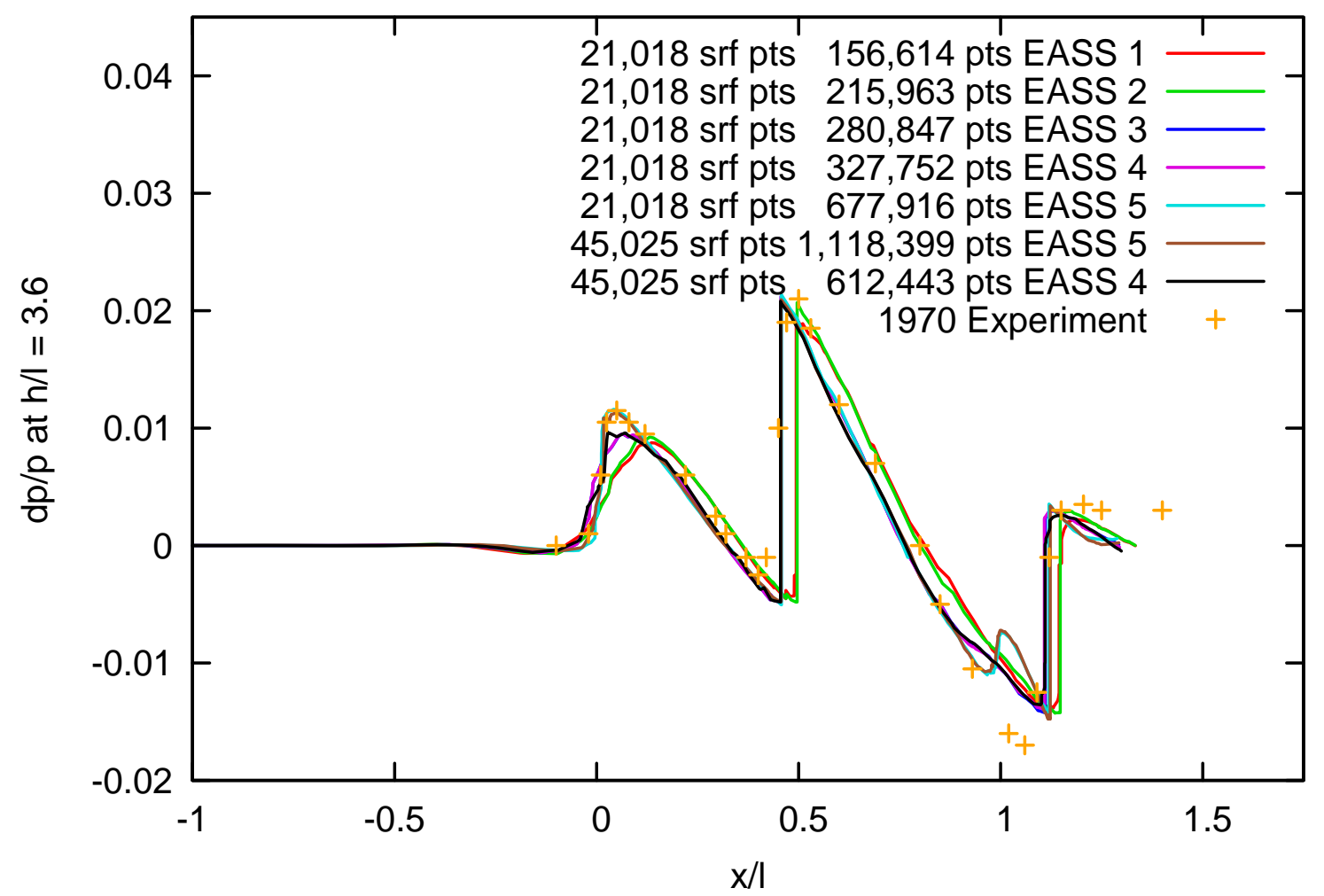

Figure 13. Delta wing-body, $M=1.68, \alpha=2.56^{\circ}, C_{L}=0.08$, AIRPLANE data extrapolated from $0.4 \mathrm{~h} / \mathrm{L}$ to $10 \mathrm{~h} / \mathrm{L}$. 